\title{
Depression: a common and burdensome complication of diabetes that warrants the continued attention of clinicians, researchers and healthcare policy makers
}

\author{
François Pouwer ${ }^{1,2}$
}

Received: 11 October 2016/Accepted: 13 October 2016/Published online: 12 November 2016

(C) Springer-Verlag Berlin Heidelberg 2016

\begin{abstract}
There is ample evidence that depression is a common comorbid health issue in people with type 1 or type 2 diabetes. Reviews have also concluded that depression in diabetes is associated with higher $\mathrm{HbA}_{1 \mathrm{c}}$ levels, less optimal self-care behaviours, lower quality of life, incident vascular complications and higher mortality rates. However, longitudinal studies into the course of depression in people with type 1 diabetes remain scarce. In this issue of Diabetologia, Kampling and colleagues (doi:10.1007/s00125-016-4123-0) report the 5 year trajectories of depression in adults with newly diagnosed type 1 diabetes (mean age, 28 years). Their baseline results showed that shortly after the diagnosis of type 1 diabetes a major depressive episode was diagnosed in approximately $6 \%$ of participants, while $8 \%$ suffered from an anxiety disorder. The longitudinal depression data showed that, in a 5 year period, $79 \%$ reported no depressive symptoms at any time, while $7 \%$ had a high depression score that improved and $14 \%$ reported worsening of depressive symptoms. Here, the clinical relevance of these findings is discussed and areas for further research are described.
\end{abstract}

Keywords Depression $\cdot$ Longitudinal $\cdot$ Psychological aspects $\cdot$ Type 1 diabetes

François Pouwer

fpouwer@health.sdu.dk

1 Department of Psychology, University of Southern Denmark, Campusvej 55, DK-5230 Odense M, Denmark

2 Department of Medical and Clinical Psychology, Centre of Research on Psychology and Somatic Diseases (CoRPS), Tilburg University, Tilburg, the Netherlands

\section{The link between diabetes and depression}

In previous decades, a large number of cross-sectional studies have investigated the prevalence of depression in people with diabetes and these studies have been included in several systematic reviews [1-4]. In the majority of these studies, depression questionnaires were used to assess depressive symptoms. Few diabetes studies have used a psychiatric diagnostic interview, which is required to diagnose a mood or anxiety disorder. Anderson et al concluded in their systematic review that in case-control studies the odds of depression were doubled compared with controls, irrespective of the type of depression assessment [1]. However, studies that determine the prevalence of depression using a self-reported measure generally report a higher prevalence of depression ('elevated depressive symptoms') than studies that use a diagnostic psychiatric interview ('diagnosed mood disorder') [1].

A recent systematic review of 14 studies in children and adolescents with type 1 diabetes concluded that clinically significant elevated depression symptoms affected $30 \%$ of participants [2]. In adults, the prevalence of depression was more than three-times higher in type 1 diabetes ( $\%$ [range]; $12 \%$ [5.8-43.3\%] vs 3.2\% [2.7-11.4\%]) and nearly twice as high in type 2 diabetes (\% [range]; $19 \%$ [6.5-33\%] vs $10.7 \%$ [3.8-19.4\%]), compared with non-diabetic controls [3]. However, an important limitation of the latter review is that studies with different assessment methods were mixed [3]. Another systematic review and meta-analysis of 12 studies also confirmed that the prevalence of depression was higher in adults with diagnosed type 2 diabetes compared with controls with normal glucose metabolism [4]. In the same review, it was found that the risk of depression was not elevated in people with undiagnosed type 2 diabetes or those with impaired glucose metabolism, compared with controls 
[4]. Following this review, the results of LifeLines, which is the largest and best study in this area so far, were published. LifeLines included $>90,000$ participants, who underwent diagnostic psychiatric interviews and measurements of fasting glucose levels [5]. In contrast with the earlier meta-analysis [3], it was concluded that both diagnosed diabetes and undiagnosed diabetes (OR 1.8 [95\% CI 1.3, 2.6]; $p=0.001$ ) were associated with a higher risk of depression [5]. Additional adjusted logistic regression analyses showed that impaired fasting glucose (prediabetes) was also associated with higher odds of depression compared with no diabetes (OR 1.2-1.6, depending on the definition of prediabetes) [5].

\section{Depression and anxiety are serious complications of type 1 diabetes}

Longitudinal data on the course of depression in diabetes remain scarce; hence the multicentre study by Kampling et al [6] is an important contribution to the literature. The authors investigated the 5-year course of depression in a relatively homogeneous group of young people (17-40 years) with a recent diagnosis $(<12$ weeks $)$ of type 1 diabetes. At baseline, $1.3 \%$ had complications (this information was obtained from their treating physicians), but it is not clear which complications were measured and how exactly. Important strengths of this study include the longitudinal design and the fact that diagnostic psychiatric interviews were used at baseline, which are the gold standard for the diagnosis of depression. Unfortunately, however, at the follow up assessments, depression was assessed by self-report only. Shortly after the diagnosis of type 1 diabetes, a major depressive episode was detected in approximately $6 \%$ of participants, while $8 \%$ suffered from an anxiety disorder (with 13\% having a mood and/or anxiety disorder). If this result is extrapolated to clinical care settings, healthcare providers can expect one in ten patients with recently diagnosed type 1 diabetes to suffer from a mood or anxiety disorder shortly after diagnosis. Thus, depression and anxiety can be regarded as common and serious complications of type 1 diabetes, including in early diagnosis.

\section{Mechanisms associated with depression and poor diabetic outcomes}

Depression has a strong negative impact on quality of life [7]. Longitudinal studies have also shown that depressed people with diabetes have a higher risk of developing microvascular and macrovascular complications and have higher mortality rates $[8,9]$. The mechanisms that link depression and poor outcomes in diabetes are still unclear and understudied, particularly in people with type 1 diabetes. On a behavioural level, different self-care behaviours might be affected by depression; a systematic review of 47 studies supported the notion that depression in diabetes was indeed associated with suboptimal self-care, with the largest negative effect being on appointment keeping. However, diet quality, medication use and glucose monitoring were also negatively affected [10]. Biological mechanisms associated with poor diabetic outcomes in depressed individuals with diabetes most likely involve poor glycaemic control, dysregulation of the hypothalamic-pituitary-adrenal (HPA) axis and low grade inflammation [8-12].

\section{Predictors of depression in diabetes}

Kampling et al showed that in a 5 year period $79 \%$ of diabetic individuals reported no depressive symptoms at any time, while $7 \%$ had a high depression score that improved and $14 \%$ reported worsening of depressive symptoms [6]. This finding is in line with results from another longitudinal study with a 3 year follow-up, examining the course (incidence, recurrence/persistence) of depressive symptoms in 2460 primary care patients with type 2 diabetes with the additional aim of identifying significant predictors of these different course patterns [13]. In this study, depression was assessed by means of a self-reported measure. Of all participants with type 2 diabetes, $26 \%$ met the criterion for depression at one or more assessments. Incident depression at follow-up was present in $14 \%$ of individuals, whilst recurrence/persistence in those with baseline depression was found in 66\% [13]. History of depression was a key clinical predictor of future depression and, once present, depression often became a recurrent condition [13].

\section{How to treat depression in diabetes}

Effective treatments for depression in diabetes include pharmacological agents and, also, psychological interventions. A meta-analysis of 14 randomised controlled trials demonstrated a large effect size for psychotherapeutic interventions that were combined with diabetes self-management training, whilst a moderate effect size was found for pharmacological interventions [14]. Two commonly used and effective psychological interventions for depression in people with diabetes include face-to-face or web-based cognitive behavioural therapy (teaching individuals with depression to think and behave differently) and mindfulness-based cognitive therapy (whereby individuals learn to observe their thoughts, feelings and actions in a mindful and non-judgemental way) $[15,16]$.

A crucial clinical question is: do these psychotherapeutic or pharmacological interventions for depression in diabetes also 
improve other health outcomes? Baumeister et al conducted a systematic review and meta-analysis of 19 randomised controlled trials, aiming to determine the effects of psychological and pharmacological interventions for depression in patients with diabetes and depression [17]. The primary outcomes were depression and glycaemic control. The authors concluded that both psychological and pharmacological interventions had a moderate but clinically significant effect on depression outcomes in people with diabetes. They also found that glycaemic control improved moderately in pharmacological trials, but the evidence was inconclusive for the impact of psychological interventions on blood glucose regulation [17]. An important limitation of this systematic review is that glycaemic control was often not the main outcome of the trials that were included. This may have resulted in floor effects (less room for improvement), since a considerable number of the participants with psychological problems had well-controlled diabetes.

\section{Can psychological interventions improve diabetic outcomes?}

A multi-site, practice-randomised controlled trial tested the hypothesis that participation in a depression management programme would contribute to a decreased risk of mortality in depressed patients with diabetes, compared with those in usual-care practices [18]. The authors concluded that, after accounting for baseline differences among patients, depressed patients with diabetes in the intervention category were less likely to have died during a 5 year follow-up, compared with those in usual care (adjusted HR 0.49 [95\% CI 0.24, 0.98]). However, Thombs and Ziegelstein [19] clearly expressed strong concerns regarding the statistical methods used in this study, which they stated would result in model overfitting. Therefore, it is currently unclear whether successful treatment of depression in people with diabetes is associated with a decreased risk of micro- and macrovascular outcomes, and lower mortality rates.

\section{How can we move forward with diabetes care?}

An important barrier to optimal diabetes care is that depression is frequently overlooked in busy clinical practices. For example, in a Dutch study among outpatients with diabetes, the frequency of recording emotional problems in patient medical charts was investigated [20]; emotional problems were recorded in only $20-25 \%$ of individuals who had moderate to severe levels of anxiety or depression [20]. Clinicians have the option use questionnaire data and/or information about the patient's history of depression to identify patients who are vulnerable to depression or experience higher levels of diabetes-related distress [13]. When diabetes healthcare teams decide to use screening tools such as the Patient Health Questionnaire-9 (PHQ-9) for depression, or the Problem Area in Diabetes Questionnaire to assess diabetes-related distress, it is important that the assessment is embedded in a stepped care approach or a collaborative care approach for depression [21]. A multicentre randomised controlled trial previously found that screening for depression in outpatients with diabetes (with written feedback to the patient and the physician, but no collaborative care approach) had minimal impact on the use of mental healthcare by outpatients and did not improve depression scores [22]. Using collaborative care approaches for depression, patients who do not respond to an antidepressant treatment are 'stepped up' from low-intensity care to a more intensive form of management [23]. Thus, repeated assessments and re-evaluations are a crucial element of collaborative care [23]. A landmark study on the effectiveness of collaborative care for depression vs usual care was conducted by Katon et al [24]. This study included a group of 214 patients with depression and poorly controlled diabetes and/or coronary heart disease from 14 primary care clinics. At 12 month follow-up, patients in the intervention group had more favourable depression scores, lower levels of $\mathrm{HbA}_{1 \mathrm{c}}$ and LDL-cholesterol, and lower systolic blood pressure compared with controls [24]. With regards to the costeffectiveness of the collaborative care intervention used, it was found that over a period of 2 years the patients in the intervention group had a mean 114 (95\% CI 79, 149) additional depression-free days and an estimated additional $0.335(95 \%$ CI $-0.18,0.85)$ quality-adjusted life years (QALYs), compared with controls [25]. The group that had received collaborative care for depression also had lower mean outpatient health costs of 594 US dollars per patient (95\% CI, $-3241,2053)$ compared with the care as usual control group [25].

The results of many of the studies on diabetes and depression that are described in this commentary are now incorporated in national and international clinical diabetes guidelines, demonstrating that these guidelines now recognise that emotional well-being is an important goal in diabetes care. These guidelines also emphasise that psychological factors, such as depression, are relevant to nearly all aspects of diabetes management and can be important barriers that hamper optimal diabetes care [26-28]. However, there is still much disparity between what the guidelines recommend and the reality of what diabetes care has to offer to people with diabetes. For example, for comprehensive care, the International Diabetes Federation (IDF) global guidelines recommend periodic psychological assessments and the inclusion of a mental health specialist to the multidisciplinary diabetes care team [26]. However, many diabetes teams who want to follow these guidelines will struggle with the 


\section{Summary Points}

1 Individuals with type 1 or type 2 diabetes have a higher risk of depression and anxiety compared with non-diabetic controls

2 Depression impacts quality of life and health outcomes (micro- and macrovascular disease and mortality) in diabetic patients. Psychological interventions may lead to improvements of these diabetic outcomes

3 A collaborative care approach via inclusion of mental health specialists in the multidisciplinary diabetes care team is recommended by clinical diabetes guidelines. However, these are not often put into practice, partly due to lack of funding

4. Further research is required to determine the course of depression in diabetes to produce guidelines for clinicians and to provide evidence for the need of psychological interventions in diabetes care

reimbursement of this type of care [29]. Additionally, funding bodies in different countries, such as the Dutch Diabetes Research Foundation, Diabetes UK and the European Federation for the Study of Diabetes (EFSD), have provided crucial support for key studies on the psychological aspects of diabetes, including depression [30]. However, a recent review of annual reports and funded-research databases of these funding agencies also showed that there were considerable differences between countries and that the proportion of funded studies with a psychosocial focus in the past five years was rather small [30]. In order to progress, a sufficient level of funding for both research into and clinical care of the psychological aspect of diabetes is essential for the necessary advancements in diabetes patient management.

\section{Conclusion}

Depression is to be regarded as a common and burdensome complication of both type 1 and type 2 diabetes that warrants the continued attention of clinicians, researchers and healthcare policy makers. A collaborative care approach, incorporating mental health specialists into diabetes care teams, is important for the emotional wellbeing of diabetic patients. However, barriers to this approach, including lack of funding, prevent this recommendation from being used in practice. Further research, similar to that by Kampling et al [6], is required to elucidate the course of depression in diabetes so that healthcare policy makers may continue to adapt guidelines to ensure optimal care to improve health outcomes in individuals with diabetes.
Duality of interest The author declares that there is no duality of interest associated with this manuscript.

Contribution statement The author was the sole contributor of this paper.

\section{References}

1. Anderson RJ, Freedland KE, Clouse RE et al (2001) The prevalence of comorbid depression in adults with diabetes: a metaanalysis. Diabetes Care 24:1069-1078

2. Buchberger B, Huppertz H, Krabbe L et al (2016) Symptoms of depression and anxiety in youth with type 1 diabetes: a systematic review and meta-analysis. Psychoneuroendocrinology 70:70-84

3. Roy T, Lloyd CE (2012) Epidemiology of depression and diabetes: a systematic review. J Affect Disord 142(Suppl):S8-S21

4. Nouwen A, Nefs G, Caramlau I et al (2011) Prevalence of depression in individuals with impaired glucose metabolism or undiagnosed diabetes: a systematic review and meta-analysis of the European Depression in Diabetes (EDID) Research Consortium. Diabetes Care 34:752-762

5. Meurs M, Roest AM, Wolffenbuttel BH et al (2016) Association of depressive and anxiety disorders with diagnosed versus undiagnosed diabetes: an epidemiological study of 90,686 participants. Psychosom Med 78:233-241

6. Kampling H, Petrak F, Farin E et al (2016) Trajectories of depression in adults with newly diagnosed type 1 diabetes: results from the German Multicenter Diabetes Cohort Study. Diabetologia. doi:10.1007/s00125-016-4123-0

7. Schram MT, Baan CA, Pouwer F (2009) Depression and quality of life in patients with diabetes: a systematic review from the European depression in diabetes (EDID) research consortium. Curr Diabetes Rev 5:112-119

8. Pouwer F, Nefs G, Nouwen A (2013) Adverse effects of depression on glycemic control and health outcomes in people with diabetes: a review. Endocrinol Metab Clin North Am 42:529-544

9. Van Dooren FE, Nefs G, Schram MT et al (2013) Depression and risk of mortality in people with diabetes mellitus: a systematic review and meta-analysis. PLoS One 8:e57058

10. Gonzalez JS, Peyrot M, McCarl LA et al (2008) Depression and diabetes treatment nonadherence: a meta-analysis. Diabetes Care 31:2398-403

11. Moulton CD, Pickup JC, Ismail K (2015) The link between depression and diabetes: the search for shared mechanisms. Lancet Diabetes Endocrinol 3:461-471

12. Lustman PJ, Anderson RJ, Freedland KE et al (2000) Depression and poor glycemic control: a meta-analytic review of the literature. Diabetes Care 23:934-942

13. Nefs G, Pouwer F, Denollet J et al (2012) The course of depressive symptoms in primary care patients with type 2 diabetes: results from the Diabetes, Depression, Type D Personality ZuidoostBrabant (DiaDDZoB) Study. Diabetologia 55:608-616

14. Van der Feltz-Cornelis CM, Nuyen J, Stoop C et al (2010) Effect of interventions for major depressive disorder and significant depressive symptoms in patients with diabetes mellitus: a systematic review and meta-analysis. Gen Hosp Psychiatry 32:380-395

15. Van Son J, Nyklícek I, Pop VJ et al (2013) The effects of a mindfulness-based intervention on emotional distress, quality of life, and $\mathrm{HbA}(1 \mathrm{c})$ in outpatients with diabetes (DiaMind): a randomized controlled trial. Diabetes Care 36:823-830

16. Van Bastelaar KM, Pouwer F, Cuijpers P et al (2011) Web-based depression treatment for type 1 and type 2 diabetic patients: a randomized, controlled trial. Diabetes Care 34:320-325 
17. Baumeister H, Hutter N, Bengel J (2014) Psychological and pharmacological interventions for depression in patients with diabetes mellitus: an abridged Cochrane review. Diabet Med 31:773-786

18. Bogner HR, Morales KH, Post EP et al (2007) Diabetes, depression, and death: a randomized controlled trial of a depression treatment program for older adults based in primary care (PROSPECT). Diabetes Care 30:3005-3010

19. Thombs BD, Ziegelstein RC (2008) Diabetes, depression, and death: a randomized controlled trial of a depression treatment program for older adults based in primary care (PROSPECT): response to Bogner et al. Diabetes Care 31, e54

20. Pouwer F, Beekman AT, Lubach C et al (2006) Nurses' recognition and registration of depression, anxiety and diabetes-specific emotional problems in outpatients with diabetes mellitus. Patient Educ Couns 60:235-240

21. Pouwer F (2009) Should we screen for emotional distress in type 2 diabetes mellitus? Nat Rev Endocrinol 5:665-671

22. Pouwer F, Tack CJ, Geelhoed-Duijvestijn PH et al (2011) Limited effect of screening for depression with written feedback in outpatients with diabetes mellitus: a randomised controlled trial. Diabetologia 54:741-748

23. Atlantis E, Fahey P, Foster J (2014) Collaborative care for comorbid depression and diabetes: a systematic review and meta-analysis. BMJ Open 4:e004706
24. Katon WJ, Lin EH, Von Korff M et al (2010) Collaborative care for patients with depression and chronic illnesses. N Engl J Med 363: 2611-2620

25. Katon W, Russo J, Lin EH et al (2012) Cost-effectiveness of a multicondition collaborative care intervention: a randomized controlled trial. Arch Gen Psychiatry 69:506-514

26. International Diabetes Federation Clinical Guideline Task Force (2012) Global guideline for type 2 diabetes. Available from www. idf.org/sites/default/files/IDF-Guideline-for-Type-2-Diabetes.pdf, accessed 11 Oct 2016

27. American Diabetes Association (2015) Standard of Medical Care in Diabetes -2015. Diabetes Care 38(Suppl 1):S15-S35

28. National Institute for Health and Care Excellence (2009) Depression in adults with a chronic physical health problem: recognition and management: clinical guidance. Available from www.nice.org. uk/guidance/cg91/chapter/1-Guidance, accessed 11 Oct 2016

29. Pozniak A, Olinger L, Shier V (2010) Physicians' perceptions of reimbursement as a barrier to comprehensive diabetes care. Am Health Drug Benefits 3:31-40

30. Jones A, Vallis M, Cooke D et al (2016) Review of research grant allocation to psychosocial studies in diabetes research. Diabet Med. doi:10.1111/dme.13255 